\title{
Safety through Collaboration: A New Challenge for Automotive Design
}

\section{Nicole Perterer}

Center for $\mathrm{HCI}$

University of Salzburg

Sigmund-Haffner-Gasse 18

Salzburg, 5020 Austria

nicole.perterer@sbg.ac.at

\section{Abstract}

A high number of car accidents are caused as a result of excessive demands and distraction. Collaboration can support drivers, since other tasks, e.g., operating with the navigation system, can be taken over by a front-seat passenger. But how can we, as researchers and HCI designers, develop and design collaborative in-car systems that really address safety issues or reduce demands during driving? Based on findings from three ethnographic studies I draw attention on the car as a safety-critical environment, where collaboration may solve safety issues.

Permission to make digital or hard copies of part or all of this work for personal or classroom use is granted without fee provided that copies are not made or distributed for profit or commercial advantage and that copies bear this notice and the full citation on the first page. Copyrights for third-party components of this work must be honored. For all other uses, contact the Owner/Author.

uses, contact the Owner/Author.
Copyright is held by the owner/author(s). CSCW' 16 Companion, February 27 - March 02, 2016, San Francisco, CA, USA

ACM 978-1-4503-3950-6/16/02

DOI: http:// 10.1145/2818052.2874344

\author{
Author Keywords \\ Collaboration; ethnographic studies; automotive;
}

\section{ACM Classification Keywords}

H.5.m. Information interfaces and presentation (e.g., $\mathrm{HCI})$ : Miscellaneous;

\section{Context and Motivation}

According to the WHO, road traffic crashes are predicted to result in the death of around 1.9 million people annually by 2020 . Road traffic injuries need to be reduced. In the field of $\mathrm{HCI}$, effective interventions may include improving safety features of vehicles and reducing negative effects of technology usage in the car. In the automotive domain, there is a high number of Advanced Driver Assistance Systems (ADAS)

available on the market that focus on safety. However, hardly any make use of the social nature and collaborative benefits of driving [4]. So far, research concerning collaboration in safety critical environments has paid much attention on industrial settings (e.g. mines), transportation (e.g., airplanes), military, or hospitals. I see the car as another example, since participating in road traffic is a dangerous endeavor.

On the one hand, collaboration in the car has the potential to reduce drivers' workload and distraction when, for instance, a front seat passenger takes over tasks like navigation. On the other hand, this 


\section{Box1. Main Research Questions}

RQ1: What collaborative practices do exist in and beyond the car? (i.e., with passengers vs. alone)

RQ2: How does the car context shape this collaboration during various contexts? (e.g., ice-glance, unfamiliar areas, commutes to work or home, traffic jam etc.)

RQ3: What are the main UX factors that have a common impact on both, collaboration between driver-passenger pairs and lone-commuters? RQ4: What are the main design challenges for developing social-sensitive and collaborative in-car systems that should be addressed? (e.g., How to achieve a common ground between the collaborators?) collaboration might lead to more distraction, when, for example, the front seat passenger uses an in-car system in a different way than intended by the driver. There is also the potential to collaborate with users outside the car, if e.g., two people, who do not sit in the same car, share their navigation task depending on contextual factors. Future ADAs that account for supporting collaboration hold potential to reduce distraction and workload and, thus can reduce traffic injuries.

How can we as researchers and $\mathrm{HCI}$ designers and in particular the CSCW community, develop collaborative and social-sensitive in-car systems that take care of safety issues or reduce workload? In order to address this challenge, we need to gain a deeper understanding of communication and collaborative practices in the car and with other users outside the car. Furthermore, related context factors (e.g., length of waiting periods) need to be taken into account. The aim of my thesis is to use collaboration to improve car safety and to introduce specific design challenges. The obtained findings from my three ethnographic studies can inform future in-car designs, which supports a more socialsensitive and collaborative usage.

\section{Related Work}

There is a notable body of research in $\mathrm{HCI}$ investigating social aspects and collaborative practices in the car through ethnographic studies (e.g.,

$[1,2,3,4,9,17])$. Specifically Oskar Juhlin's group (e.g., [7] conducted various ethnographic studies that served as a basis for a range of systems related to the social dimensions of driving (e.g., the SoundPryer system [12]). Contemporary research also focuses on driverpassenger pair cooperation in navigational tasks $[4,17]$
Regarding observable collaborative practices, Gärtner et al. [5] highlighted that drivers and passengers often experience problems in effectively communicating with one another. Human-human mediated communication beyond the car has become more important in the automotive domain in the last years [e.g., 16, 18]. Lamas et al. [8] did some basic research and explored user requirements for a driver-to-driver communication device within a cross-cultural study. Furthermore, Cycil et al. [3] looked at how passengers' assistance during mobile telephone calls influences driving while being engaged in co-located and remote conversations. Gauglitz et al. [6] present a system that supports an augmented shared visual space for live mobile remote collaboration on physical tasks. Nevertheless, the systems and applications are mainly designed for static settings as well as do not take specific context factors into account.

To inform the design of future collaborative in-car systems it is necessary to understand the context of use holistically, which means to include all influencing factors such as environmental conditions, traffic conditions, constraints due to car properties or internal social factors [10]. Accordingly, four research questions were defined as shown in Box 1.

\section{Work in Progress and Results to Date}

Study I: The goal of my first ethnographic study was to gain an in-depth understanding of collaboration in the car context in general and of how technology of how technology supports these interactions (e.g., collaboration). The study, which was published at CSCW 2013 [15], revealed that collaborative navigation between driver-passenger pairs is of high importance for driving, especially at night. Customized navigation 
devices supported this collaboration by providing different information for the driver and passenger. I further presented a design approach for future collaborative navigation devices [13].

Study II: Taking collaborative aspects outside the car into account, I carried out a second ethnographic study with 13 single-person private car-commuters. Since traffic jams include several waiting periods that could be used for such collaboration, my idea was to research and analyze these specific waiting periods in terms of their collaborative potential. (planned publication at MobileHCI 2016). Results show that lone-commuters took advantage of short waiting periods for collaborative practices (e.g., coordinating to-do list). Moreover, the daily 'fighting' of mileage was transferred into collaborative gaming for fuel saving or to have fun. This obtained knowledge served as a base for a design workshop with eight $\mathrm{HCI}$ experts that resulted in 32 design ideas [11].

Study III: As my previous research has shown [15], many in-car assistance situations can be related to collaborative navigation or support in demand situations. In order to help the driver in navigation and driving related tasks, I created a tablet based navigation app, especially designed for front-seat passengers. To evaluate the collaborative potential of my prototype, I investigated navigation practices between familiar individuals (i.e., couples) in real driving situations. The prototype was presented at this year's conference on Automotive User Interface [14]. The data shows that basic information such as turn-byturn instructions should be given to both, the driver and the front-seat passenger to generate a common basic understanding.

\section{Expected Contribution}

This research will result in two primary contributions. First, this work will expand current research on in-car collaboration as well as beyond the car. Second, this works enables deeper understanding of potentials and design challenges as well as provide suggestions for developing social-sensitive and collaborative in-car designs. For instance, how to achieve a common ground for drivers and front-seat passengers and other people outside the car. With my thesis I provide a basis for reconsidering human-human-mediated interaction in a safety-critical environment such as the car to allow for more collaboration while driving.

\section{Objectives for the CSCW DC}

The colloquium would be the ideal platform to present and discuss my ongoing research in a professional atmosphere, and to obtain valuable feedback from fellow attendees, senior researches and experts in the field. I would also welcome a discussion on the effective evaluation of combined video, interview and GPS data (used in Study II).

\section{Acknowledgements}

Thanks to my supervisor, Prof. Manfred Tscheligi for his guidance and also thanks to the financial support by the Austrian Federal Ministry of Science Research and Economy and the National Foundation for Research, Technology and Development and AUDIO MOBIL Elektronik GmbH (Christian Doppler Laboratory for 'Contextual Interfaces').

\section{References}

1. Barry Brown and Eric Laurier. 2012. The normal natural troubles of driving with GPS. In Proceedings 
of the SIGCHI Conference on Human Factors in Computing Systems (CHI '12), 1621-1630.

2. Barry Brown et al. Driving and 'Passengering': Notes on the Ordinary Organization of Car Travel. Mobilities 3, 1 (January 2008), 1-23.

3. Chandrika Cycil et al. 2013. 'Eyes free' in-car assistance: parent and child passenger collaboration during phone calls. In Proceedings of the International Conference on Human-computer Interaction with Mobile Devices and Services (MobileHCI '13), 332-341.

4. Jodi Forlizzi, William C. Barley, and Thomas Seder. 2010. Where should I turn: moving from individual to collaborative navigation strategies to inform the interaction design of future navigation systems. In Proceedings of the SIGCHI Conference on Human Factors in Computing Systems (CHI '10), 12611270.

5. Magadalena Gartner et al. 2014. "Dad, Stop Crashing My Car!": Making Use of Probing to Inspire the Design of Future In-Car Interfaces. In Proceedings of the International Conference on AutomotiveUI (AutomotiveUI '14), 27,8.

6. Steffen Gauglitz et al. 2014. World-stabilized annotations and virtual scene navigation for remote collaboration. In Proceedings of the Annual ACM symposium on User Interface Software and Technology (UIST '14), 449-459.

7. Oskar Juhlin. 2010 Social Media on the Road -The future of car based computing, Monography. Springer Verlag, London.

8. Raphael Lamas et al. 2014. A Driver-to-Driver Communication Device to Improve Drivers' Interaction. In Adjunct Proceedings of the International Conference on AutomotiveUI (AutomotiveUI '14), 1-3.

9. Gilly Leshed et al. 2008. In-car gps navigation: engagement with and disengagement from the environment. In Proceedings of the SIGCHI
Conference on Human Factors in Computing Systems (CHI '08), 1675-1684.

10. Alexander Meschtscherjakov et al. 2011. Capture the car!: qualitative in-situ methods to grasp the automotive context. In Proceedings of the International Conference on AutomotiveUI (AutomotiveUI '11), 105-112.

11. Marianne Obrist et al. 2013. Experiential Perspectives on Road Congestions. In Adjunct Proceedings of the SIGCHI Conference on Human Factors in Computing Systems (CHI EA '13), 535540.

12. Mattias Östergren and Oskar Juhlin. 2004 Sound Pryer: truly mobile joint music listening. International Workshop on Mobile Music Technology, Göteborg, Schweden.

13. Nicole Perterer (*Gridling), Alexander Meschtscherjakov and Manfred Tscheligi 2012. I need help! Exploring collaboration in the car. In Proceedings of the ACM 2012 Conference on Computer Supported Cooperative Work Companion (CSCW '12), 87-90.

14. Nicole Perterer, Alexander Meschtscherjakov and Manfred Tscheligi. 2015. Co-Navigator: an advanced navigation system for front-seat passengers. In Proceedings of the International Conference on AutomotiveUI(AutomotiveUI '15), 187-194.

15. Nicole Perterer et al. 2013. Come drive with me: an ethnographic study of driver-passenger pairs to inform future in-car assistance. In Proceedings of the 2013 conference on Computer supported cooperative work (CSCW '13). 1539-1548.

16. Sandra Trösterer et al. 2015. Four Eyes See More than Two: Shared Gaze in the Car. In: J. Abascal et al. (Eds.): INTERACT 2015, Part II, 331- 348.

17. Mimi Sheller and John Urry The city and the car. IJUR 24, 4 (2000), 737-757. 